\title{
A Computação como uma Ferramenta na Avaliação do Comportamento de Ligas Metálicas Submetidas ao Processo de Soldagem
}

\author{
The Computation as a Tool for Evaluation of the Behavior of \\ Metallic Alloys Submitted to the Welding Process \\ Professor Doutor Carlos Roberto Xavier (Engenharia Mecânica - Unifoa) \\ crxavier@yahoo.com.br / carlos.xavier@foa.org.br \\ Professor Mestre Alexandre Fernandes Habibe (Engenharia Mecânica - Unifoa) \\ alexandre.habibe@,foa.org.br \\ Professor Doutor José Adilson de Castro (Universidade Federal Fluminense) \\ adilson@metal.eeimvr.uff.br
}

\section{Resumo}

A soldagem tem um papel de destaque dentre os processos industriais que visam à transformação do aço em produtos acabados, não se limitando somente ao campo da fabricação metálica, mas sendo amplamente utilizada também em segmentos como manutenção industrial e na aplicação de revestimentos protetores sobre superfícies metálicas com a finalidade de lhes conferir certas características especiais como resistência à corrosão e ao desgaste. Pode-se citar a utilização da soldagem por áreas de tecnologia de ponta como as indústrias aeroespacial, automobilística e naval. Dessa forma, muitas pesquisas têm sido realizadas objetivando a um melhor entendimento dos complexos fenômenos envolvidos durante o procedimento de soldagem e como estes afetam as propriedades do metal soldado, a fim de se prever o seu comportamento quando em uso. Neste trabalho é fornecido um panorama do estágio atual da soldagem, focando principalmente no uso da computação, dentre outras técnicas utilizadas, como uma ferramenta para a melhor compreensão dos fenômenos que ocorrem durante o processo e que afetam as propriedades tecnológicas do metal.

Palavras-chave: Computação, Soldagem, Propriedades, Ligas Metálicas.

\begin{abstract}
Welding plays an important role among the industrial processes that aims at the steel transformation in finished products, not only limiting to the field of the
\end{abstract}


metallic manufacture, but also being widely used in segments as industrial maintenance and the protective covering application on metallic surfaces with the purpose of confer to them special characteristics as resistance to the corrosion and the wear. Some areas that require high technologies can be mentioned as those where welding has detached application, as for example aerospace, automotive and shipbuilding industries. Thus, researches has been carried out in order to obtain a better understanding with regard to the complex phenomena involved during the welding procedure and how it can affects the welded metal properties in order to foresee its behavior when in use. In this work, an overview of the welding current stage is supplied, focusing mainly on the use of the computation, among others techniques, as a tool for better understanding of the phenomena that occur during the process and affect the technological properties of the metal.

Key Words: Computation, Welding, Metallic Alloys, Properties.

\section{O Uso da Computação na Soldagem}

Alguns autores ${ }^{1-4}$ têm usado a simulação computacional como uma importante ferramenta na obtenção de informações detalhadas sobre as características da soldagem e a influência dos parâmetros sobre as mesmas.

De acordo com Zacharia et al. ${ }^{1}$, a soldagem tem evoluído nas últimas poucas décadas de uma arte empírica para uma atividade, incorporando as mais avançadas ferramentas das várias ciências básicas e aplicadas, sendo que um progresso significativo tem sido alcançado no entendimento do processo e sobre os materiais soldados. Em função de um grande número de variáveis envolvidas, o que gera uma grande base de dados experimental, o entendimento e o controle do processo de soldagem tornam-se caros e demorados ou, até mesmo, impraticáveis. Dessa forma, um importante recurso é a simulação computacional da soldagem através do estabelecimento de um conjunto de equações representando os processos físicos essenciais da soldagem, sendo que os resultados obtidos a partir dos modelos fenomenológicos dependem primordialmente da qualidade das relações físicas contidas nos modelos e da fidedignidade dos dados fornecidos aos mesmos.

Pode ser visto na Figura 1 o fluxo convectivo em uma poça de solda estacionária como um resultado de uma complexa interação entre as várias forças motrizes. 


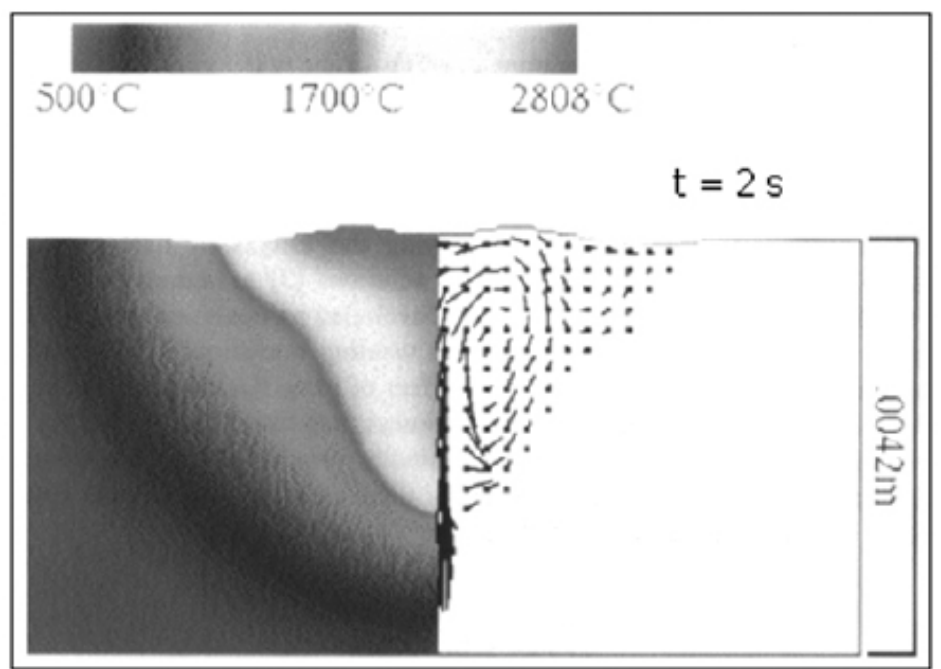

Figura 1 - Simulação computacional do fluxo de calor e de fluido na poça de solda.

Figura 1 - Simulação computacional do fluxo de calor e de fluido na poça de solda ${ }^{1}$.

Ronda e Oliver ${ }^{2}$ propuseram um modelo termo-mecânico-metalúrgico (TMM) para a soldagem de aços. Segundo esses autores, um número de fenômenos ocorre na ZAC, sendo os mais importantes os relacionados ao aquecimento e resfriamento, à dilatação térmica, às deformações elásticas e inelásticas, à solidificação da poça de fusão, à transformação de fases no estado sólido e às transformações induzidas por plasticidade acompanhadas por efeitos de deformação volumétrica, sendo que o estado do material no processo TMM pode ser definido por quatro variáveis constitutivas, que são a taxa de deformação, a tensão, a temperatura e a fração de fases.

Os autores ${ }^{2}$ mencionaram que as variáveis de estado são acopladas e o acoplamento é fornecido por um sistema de equações expressando o balanço de trabalho virtual, o balanço de energia interna, e as leis para evolução das frações dos constituintes (Tabela 1), sendo que a reação do material soldado durante o processo TMM é determinada pela transformação microestrutural, parâmetros de endurecimento, equações constitutivas para os processos termo-eláticos, teoria da plasticidade clássica e plasticidade induzida por transformação.

Tabela 1 - Leis para evolução das transformações ${ }^{2}$.

\section{LEI}

Johnson- Mehl -Avrami (transformação difusional)
FRAÇÃO RELACIONADO

Ferrita ou Perlita

Taxa de tensão interna, Taxa de temperatura, 
Koistinen-Marburger

(estendido para

transformação difusional

modificado por pressão e

tensão)
Generalização tri-dimensional da estatística termodinâmica
Martensita

Taxa de tensão

equivalente, Taxa de

temperatura,
Martensita
Taxa de energia de deformação, Taxa de temperatura.

A transformação bainítica é considerada dependente tanto de processos difusivos (formação da ferrita e da perlita) quanto de processos que não envolvem a difusão (reação martensítica).

Pode ser visto nas figuras 2 a 4 o resultado da simulação computacional referente à soldagem de topo de duas placas de aço simétricas de dimensões $100 \times 50$ × $20 \mathrm{~mm}$, efetuando-se um total de três passes de soldagem através do processo MIG-Plasma.

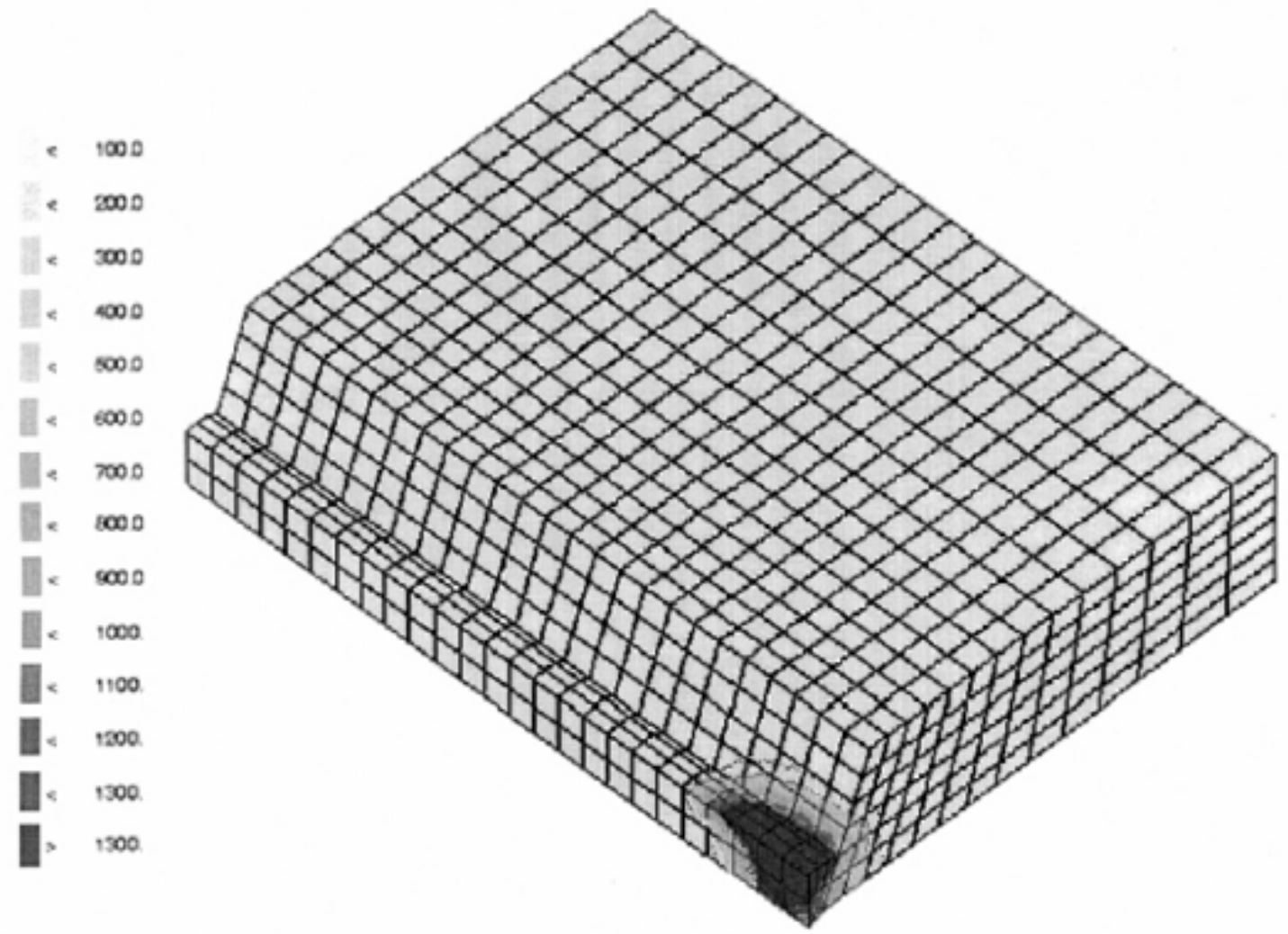

Figura 2 - Campo de temperatura durante o primeiro passe de soldagem de um total de três ${ }^{2}$. 


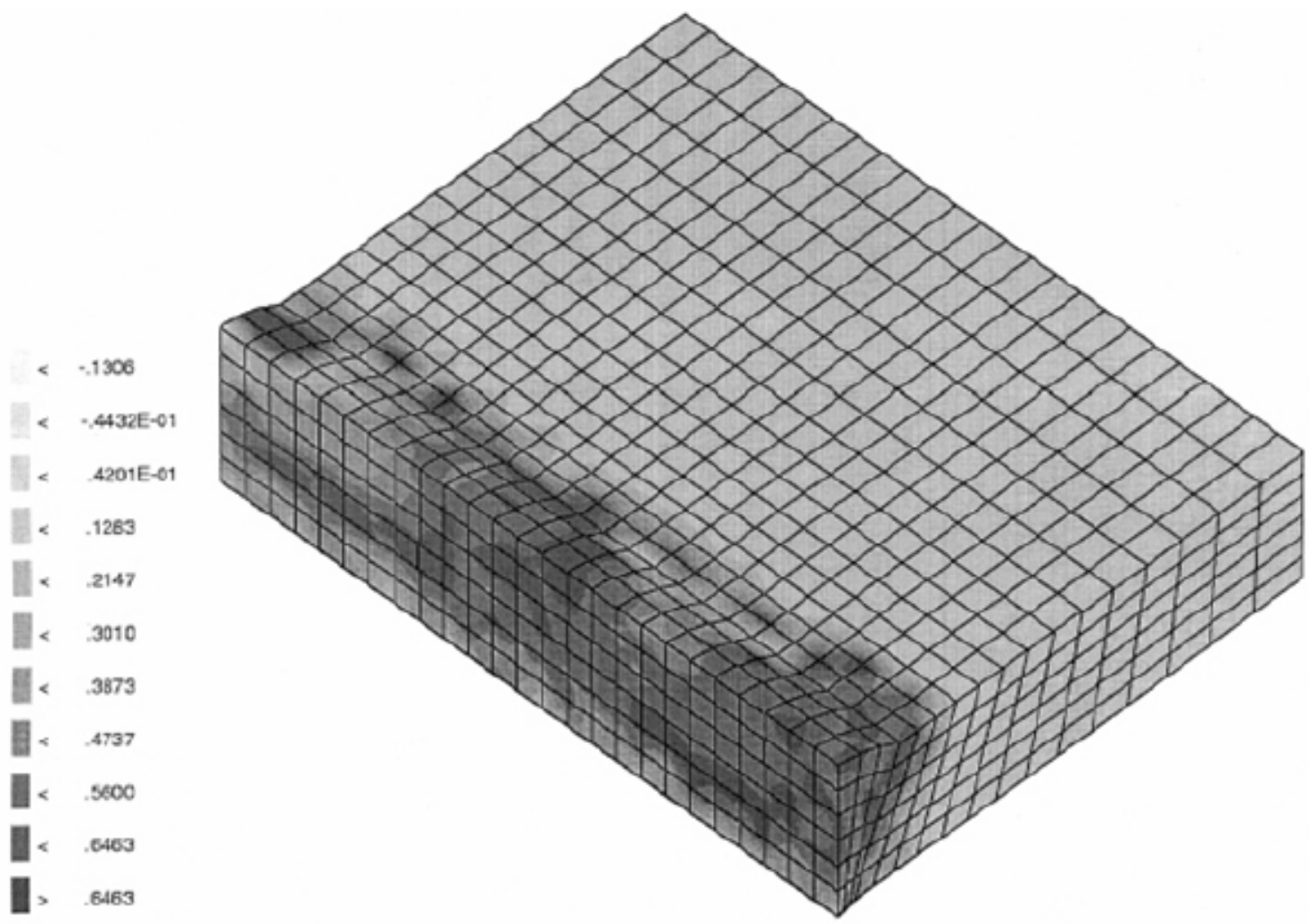

Figura 3 - Fração volumétrica de bainita após três passes de soldagem².

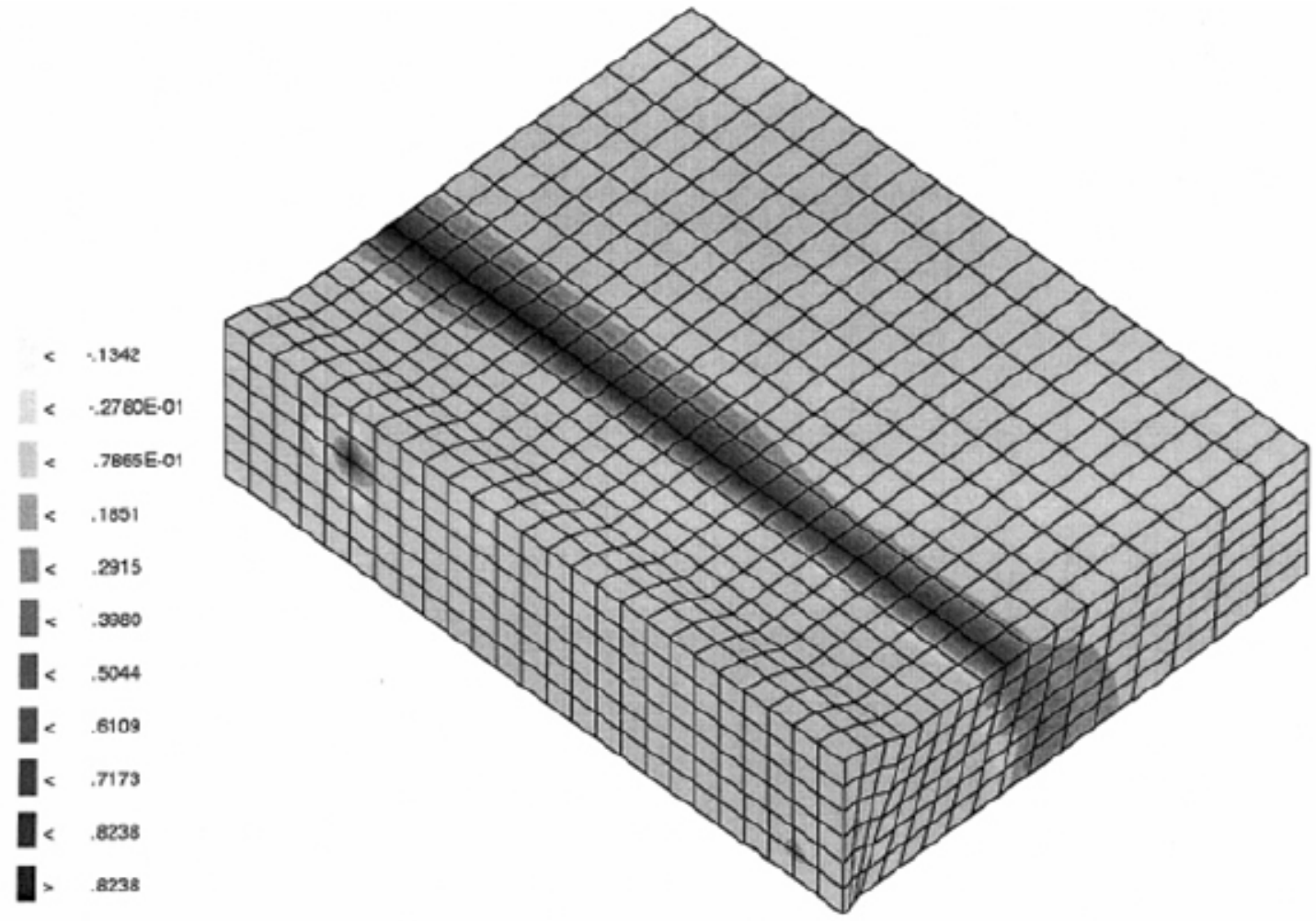

Figura 4 - Fração volumétrica de martensita após três passes de soldagem². 
Zhang et al. ${ }^{3}$ mencionaram que o entendimento da evolução microestrutural na soldagem tem tido um importante destaque nas pesquisas contemporâneas, já que as propriedades do material são afetadas pela sua microestrutura, sendo que a principal tarefa para se atingir esse objetivo é desenvolver o entendimento quantitativo da cinética de transformação das várias fases sob rápidas condições de aquecimento e resfriamento, as quais ocorrem durante a soldagem das ligas metálicas.

Os autores ${ }^{3}$ utilizaram, no desenvolvimento do trabalho, um aço carbono comum para estudar a cinética de transformação de fases na ZAC durante o aquecimento na soldagem pelo processo TIG. Foi avaliada a transformação de ferrita para austenita, utilizando-se para isso um método experimental baseado na técnica de mapeamento por difração de raios X combinado com o modelamento matemático. $\mathrm{O}$ modelamento consistiu de equações para fluxo de fluido e transferência de calor em três dimensões e da equação de Johnson-MehlAvrami modificada para a transformação não-isotérmica de ferrita para austenita (Equação $1)$.

$$
f=1-\exp \left[-\left\{\sum_{i=1}^{m} k_{0} \exp \left(-\frac{Q}{R T_{i}}\right) \Delta t\right\}\right]
$$

onde $f$ é a fração transformada da austenita, $Q$ é a energia de ativação para transformação ferrita-austenita, incluindo a nucleação e crescimento da austenita, $n$ é o expoente de Johnson-Mehl-Avrami, $k_{0}$ uma constante, $R$ é a constante universal dos gases, $\Delta t$ o intervalo de tempo, $T_{i}$ a temperatura no i-ésimo passo no tempo e $m$ o número total de passos discretos no tempo.

Segundo Tsirkas et al. ${ }^{4}$, a utilização de tecnologias de soldagem de alta densidade de potência, tal como a soldagem a laser, tem aumentado consideravelmente na manufatura industrial. Não obstante, complexos fenômenos ocorrem durante a soldagem a laser, sendo que os métodos tradicionais de análise, fundamentados em experimentos, têm encontrado muitas dificuldades no fornecimento de informações a fim de aperfeiçoar esse processo.

Com a finalidade de estender a aplicação industrial da soldagem a laser e tornar o processo mais confiável, é necessário o desenvolvimento de técnicas de controle apropriadas. Dessa forma, a simulação numérica dos processos de soldagem tem sido um tópico principal das pesquisas nos últimos anos, sendo que os resultados da simulação podem ser usados para explicar a essência física de alguns fenômenos complexos e podem ser usados também como a base para a otimização dos parâmetros envolvidos nos processos de soldagem. 
Os autores ${ }^{4}$ tomaram como base a lei de Johnson-Mehl-Avrami (Equação 2) nas transformações metalúrgicas envolvendo difusão do aço estudado, ou seja, as transformações austenítica, ferrítica, perlítica e bainítica.

$p(T, t)=\bar{p}(T)\left\{1-\exp \left[-\left(\frac{t}{\sigma_{R}(T)}\right)^{n(T)}\right]\right\}$

(2)

onde $p$ representa a proporção de fase obtida após um tempo infinito na temperatuta $T, \tau{ }_{\mathrm{R}}$ é o tempo de retardo e n é o expoente associado à taxa de reação, sendo que os parâmetros da Equação 2 foram extraídos do diagrama de transformação no resfriamento contínuo correspondente ao aço estudado.

Para o caso da transformação martensítica adotou-se uma equação baseada na lei de Koistinen-Marburger (Equação 3).

$p_{m}(T)=\bar{p}_{m}\left\{1-\exp \left[-b\left(M_{S}-T\right)\right]\right\}$

onde $p_{m}$ representa a proporção da fase obtida em uma temperatura infinitamente baixa e $M_{s}$ e $b$ representam, respectivamente, a temperatura inicial de transformação e a evolução dos processos de transformação de acordo com a temperatura ${ }^{4}$.

Xavier ${ }^{5}$ utilizou a simulação computacional a fimm de prever os campos de temperatura e transformação de fases quando da deposição de cordão de solda através do processo MIG sobre substratos de aço AISI-4340. Dentre as equações utilizadas no desenvolvimento do seu trabalho estão as equações da energia (Equação 4) e da cinética de formação das fases (Equações 5 e 6) que foram resolvidas, de forma acoplada, através do método dos volumes finitos.

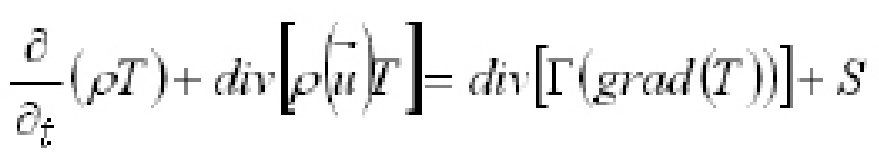

(4)

onde T é a difusividade e $S$ o termo fonte. 
$\frac{d y_{j}}{d t}=\left(1-y_{1}-y_{2}, \ldots, y_{n}\right) \frac{d B_{i} t^{\rho p t}}{d t} \quad i=1,2, \ldots, n$

(5)

onde,

- $y_{1}, y_{2}, \ldots \ldots, y_{n}$ correspondem às frações volumétricas das fases;

- $m$ é o coeficiente de Avrami;

- $B_{i}=B_{i}(T)$ são parâmetros caracterizando as taxas de nucleação e processos de crescimento (obtidos das curvas TTT), os quais são redefinidos para se levar em consideração também o efeito do tamanho de grão austenítico nas transformações.

$f=1-\exp \left(\_0,011\left(M_{s-} T\right)\right)$

(6)

onde $f$ corresponde à fração volumétrica da martensita formada e $M_{s}$ é à temperatura de início de sua transformação (obtida da curva TTT)

Alguns resultados obtidos para este trabalho podem ser vistos nas figuras 5 e 6 , que correspondem respectivamente à distribuição de temperatura e a formação do constituinte martensita na velocidade de soldagem de $35 \mathrm{~cm} / \mathrm{min}$.

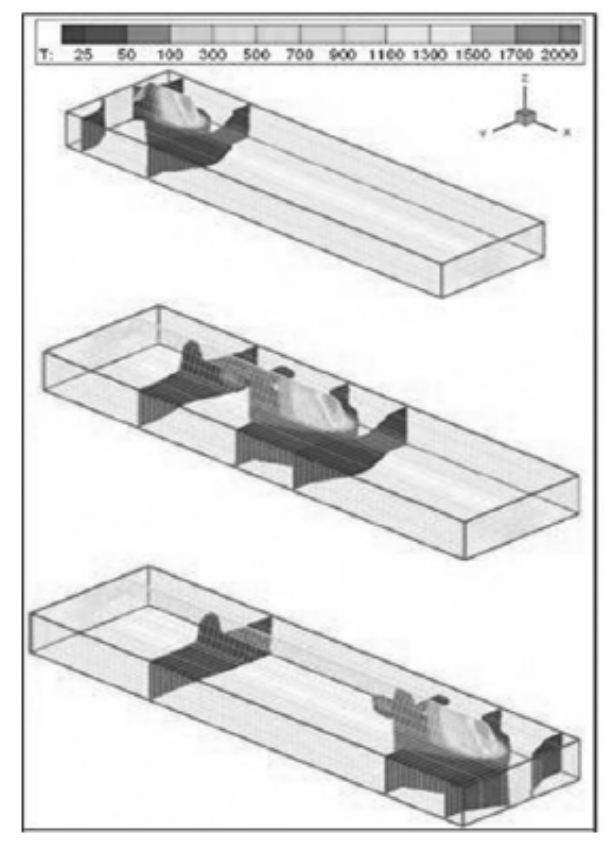

Figura 5 - Simulação computacional do cordão de solda e distribuição da temperatura (Vel.: $35 \mathrm{~cm} / \mathrm{min})^{5}$. 


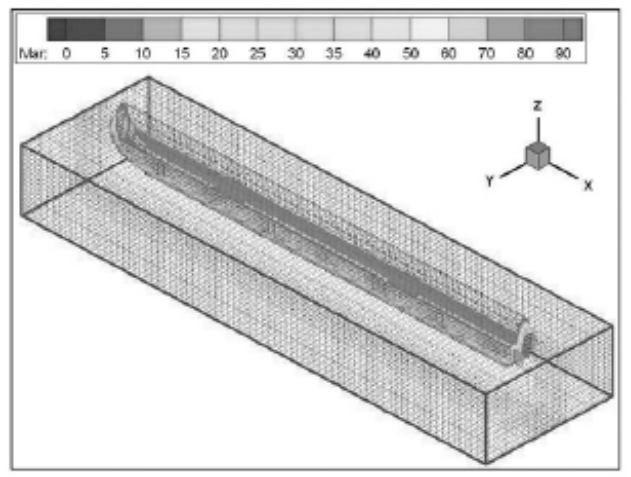

Figura 6 - Simulação computacional da fração volumétrica de martensita após a deposição do cordão de solda $(\mathrm{V}=35 \mathrm{~cm} / \mathrm{min})^{5}$.

\section{Conclusão}

A simulação computacional tem se mostrado uma ferramenta apropriada e de grande importância na análise fenomenológica e na previsão das transformações mecânicas e metalúrgicas sofridas pelas ligas metálicas quando submetidas a um determinado procedimento de soldagem podendo-se, a partir destas considerações, tentar prever o comportamento em serviço de um determinado componente mecânico.

Deve-se salientar que a obtenção de resultados realísticos é inerente a um modelamento que leve em consideração todos os fenômenos envolvidos no processo, o que no caso da soldagem não é uma tarefa simples devido à grande quantidade de variáveis físico-químicas e parâmetros a serem considerados.

Um critério importante é a confrontação dos resultados obtidos com resultados experimentais a fim de validar o modelo utilizado. Para isso, faz-se necessário a utilização de aparatos experimentais para a realização de ensaios em condições controladas que possam ser simuladas e que forneçam informações e dados fidedignos para a comparação. Dessa forma, a simulação pode se tornar uma ferramenta com grande potencial na previsão das transformações que ocorrem em metais e ligas a serem soldados utilizando-se diferentes processos e sob as mais variadas circunstâncias.

Vale ressaltar que os modelos após validados fornecem parâmetros que não são acessíveis através de experimentos. Os modelos desenvolvidos podem então ser utilizados para prever o processo em escala real e fornecer condições de trabalho que não são facilmente monitorados ou são de elevado custo. Portanto, a simulação computacional é uma ferramenta de grande utilidade com baixo custo.

\section{Referências Bibliográficas}


1. ZACHARIA, T., VITEK, J. M., GOLDAK, J. A., DEBROY, T. A., RAPPAS, M., BHADESHIA, H. K. D. H. Modeling of Fundamental Phenomena in Welds. Modelling and Simulation in Materials Science and Engineering, v. 3, n. 2, p. 265-288, Mar. 1995.

2. RONDA, J., OLIVIER, G. J. Consistent Thermo-Mechano-Metallurgical Model of Welded Steel With Unified Approach to Derivation of Phase Evolution Laws and Transformation-Induced Plasticity. Computer Methods in Applied Mechanics and Engineering, v. 189, n. 2, p. 361-417, Sep. 2000.

3. ZHANG, W., ELMER, J. W., DEBROY, T. Modeling and real time mapping of phases during GTA welding of 1005 steel. Materials Science and Engineering A, v. A333, p. 320-335, Aug. 2002.

4. TSIRKAS, S. A., PAPANIKOS, P., KERMANIDIS, TH. Numerical simulation of the laser welding process in butt-joint specimens. Journal of Materials Processing Technology, v. 134, p. 59-69, Mar. 2003.

5. XAVIER, C. R., Modelamento e Simulação de Temperatura e Transformação de Fases na Soldagem MIG de Aços: 2006, Tese (Doutorado) - Pós-Graduação em Engenharia Metalúrgica, Universidade Federal Fluminense, Volta Redonda.

\section{Informações bibliográficas:}

Conforme a NBR 6023:2002 da Associação Brasileira de Normas Técnicas (ABNT), este texto científico publicado em periódico eletrônico deve ser citado da seguinte forma: XAVIER, C. R; HABIBE, A. F; CASTRO, J. A Computação como uma Ferramenta na Avaliação do Comportamento de Ligas Metálicas Submetidas ao Processo de Soldagem. Cadernos UniFOA, Volta Redonda, ano 2, n. 3, mar. 2007. Disponível em:

$<$ http://www.unifoa.edu.br/pesquisa/caderno/edicao/03/13.pdf $>$ 